\title{
MALNUTRICIÓN POR EXCESO Y RIESGO \\ CARDIOMETABÓLICO EN ESCOLARES DE SEGUNDO Y TERCERO MEDIO DE LA COMUNA DE LOTA DE CHILE
}

\author{
MALNUTRITION BY EXCESS AND \\ CARDIOMETABOLIC RISK IN SECOND AND THIRD \\ GRADE CHILDREN OF LOTA COUNTY OF CHILE
}

\author{
Kristian Buhring B. (1), Patricio Oliva M. (2), \\ Claudia Villablanca A. (3), Valeria Rifo M. (3) \\ (1) Departamento de Salud Pública, Facultad de Medicina, \\ Universidad Católica de la Santísima Concepción. Concepción, Chile. \\ (2) Facultad de Odontología, Universidad del Desarrollo, Concepción, Chile. \\ (3) Carrera de Nutrición y Dietética, Facultad de Medicina, \\ Universidad Católica de la Santísima Concepción. Concepción, Chile.
}

\begin{abstract}
Adolescent overweight is a widespread problem which increases the risk of developing metabolic diseases. The objective of this study was to associate metabolic syndrome and cardiovascular risk in a school in the district of Lota. A cross-sectional study with simple random probability sampling in 286 children, 14 and 18 years was taken. Prevalence of malnutrition was established, and Pearson correlation was performed. The correlations were: weight and height, 0.55; weight and waist circumference 0.87; weight and body mass index 0.86; height and waist circumference 0.39; height and body mass index 0.047; body mass index and waist circumference 0.80 . The presence of the metabolic syndrome conditions the presence of nutritional diseases, meaning that anthropometric indicators may be suitable for identifying the presence of cardiometabolic risk in adolescents.
\end{abstract}

Key words: Obesity; overweight; cardiometabolic risk; school; waist circumference.

Este trabajo fue recibido el 13 de Abril de 2010, aceptado con modificaciones el 26 de Octubre de 2010 y aceptado para ser publicado el 10 de Octubre de 2011.

\section{INTRODUCCIÓN}

Los adolescentes forman un grupo de gran vulnerabilidad, desde los puntos de vista social, psicológico, educacional, de salud y nutricional. Definida por la OMS dentro de un rango etario que abarca desde los 10 a los 19 años (1) constituye una etapa donde biológicamente el individuo progresa desde la aparición inicial de las características sexuales secundarios hasta la madurez sexual, y socialmente se realiza una transición del estado de dependencia socioeconómica total a una relativa independencia (2).

La edad de inicio de este periodo presenta una variabilidad individual, dependiendo de la interacción de factores genéticos con el ambiente nutricional, psicosocial y climático (3). Los adolescentes corresponden en Chile al 17,9\% de la población (4), Siendo un grupo que presenta características epidemiológicas propias que necesitan de un abordaje sistemático, en problemas tales como la malnutrición por exceso. La malnutrición por exceso está presente en un $33 \%$ de los adolescentes (5), porcentaje que sigue en aumento.

Se ha visto en adultos que la medición de la cantidad de grasa abdominal se relaciona con un aumento en el riesgo de desarrollar enfermedades metabólicas tales como, diabetes mellitus tipo 2, la hipertensión arterial, 
dislipidemias entre otras (6). En el último tiempo se ha investigado la relación existente entre la distribución de la grasa corporal y el riesgo de desarrollar enfermedades metabólicas en niños y adolescentes, ya que la circunferencia de la cintura es un buen predictor de las complicaciones metabólicas y de riesgo cardiovascular en este grupo etario $(7,8)$. Esta medición presenta utilidad desde el punto de vista epidemiológico y clínico, ya que es utilizada en el diagnóstico del síndrome metabólico, el cual se diagnostica por la presencia de tres de los cinco parámetros siguientes: la circunferencia de cintura $\geq$ percentil 90 , la presión arterial $\geq$ percentil 90 para la edad, HDL $\leq 40 \mathrm{mg} / \mathrm{dl}$, triglicéridos $\geq 110 \mathrm{mg} /$ dl y glicemia $\geq 110 \mathrm{mg} / \mathrm{dl}$ en ayunas (9).

A pesar de la importancia que este grupo etario representa en la población, los organismos de salud gubernamentales no han puesto en marcha a nivel nacional un programa que se adecue a las necesidades del grupo en cuestión, ya que el programa que actualmente utilizan abarca sólo a los adolescentes desde un punto de vista psicosocial, poniendo énfasis en aspectos como prevención de embarazos no deseados, evitar el consumo de drogas y alcohol, entre otros aspectos.

Por lo cual, el objetivo de esta investigación fue asociar el síndrome metabólico y el riesgo cardiovascular en alumnos de enseñanza media en la comuna de Lota.

\section{SUJETOS Y MÉTODOS}

El diseño del estudio es de corte transversal. El muestreo de carácter probabilístico, de tipo aleatorio simple, polietápico, con un universo de 1.116 alumnos, del cual surge una muestra de 286 niños de la comuna de Lota, de ambos sexos, de edades que fluctuaban entre los 14 y 18 años, pertenecientes al segundo y tercero medio, con un error alfa del 5\%, una confiabilidad del $95 \%$ y un nivel de heterogeneidad del $50 \%$. Se aseguró que la extracción de los casos de distribuyera homogéneamente en la población.
La recopilación de la información se realizó en las siguientes etapas:

- Etapa 1: Evaluación de patrones alimentarios y de actividad física, a través de la aplicación de una encuesta basada en la Guía para una vida saludable parte 1 (10).

- Etapa 2: Evaluación antropométrica donde se evaluó peso, talla y circunferencia de cintura y posteriormente se realizó el diagnóstico nutricional (11) de cada uno de los participantes.

Los registros y mediciones se realizaron con procedimientos estandarizados y fueron determinados por los investigadores quienes estaban entrenados para estos procedimientos.

Se consideró obeso a los niños que presentaron un IMC/E mayor al percentil 95 y riesgo de obesidad entre los percentiles 85 a 95 según la tabla del Center for Disease Control (CDC) de la OMS para niños de 6 a 18 años (12).

La evaluación de la circunferencia de cintura se realizó en el punto medio entre la última costilla y la cresta ilíaca, se consideró aumentada cuando fue mayor o igual al percentil 90 según las tablas circunferencia de cintura de niños, niñas y adolescentes (13).

Se estableció en primer lugar la prevalencia de malnutrición, y posteriormente se analizaron los resultados mediante la correlación de Pearson, analizados estadísticamente a través del programa R- Project $C$.

\section{RESULTADOS}

El análisis descriptivo de los resultados de la población estudiada indica que el porcentaje de malnutrición por exceso entre los escolares fue $29 \%$ (tabla 1), que se distribuyen entre los diagnósticos antropométricos de riesgo de obesidad (21\%) y obesidad (8\%).

A pesar de que $69 \%$ de los escolares presentaban un estado nutricional por antropometría normal, hay que considerar que una tercera parte de la muestra tuvo una

\section{TABLA 1}

\section{Clasificación nutricional antropométrica de los escolares.}

\section{Dg. antropométrico}

Bajo peso (BP)

Normal (N)

Riesgo de obesidad (RO)

Obesidad (OB)

Total
Frecuencia

6

197

61

22

286
Porcentaje

$2 \%$

$69 \%$

$21 \%$

$8 \%$

$100 \%$ 
clasificación que la situó en riesgo para desenlaces de eventos agudos y crónicos.

\section{Estudio de correlaciones}

Al evaluar correlacionalmente las variables que presentan una alta correspondencia entre sí, se encuentran las variables de peso, circunferencia de cintura e índice de masa corporal se relacionan con variables tales como hábitos alimentarios, se excluye la talla y edad. Aun cuando la talla sea indispensable para el cálculo del índice de masa corporal, la correlación entre ambas es débil, lo que indica una mayor importancia del peso frente a la talla en el cálculo del índice de masa corporal. Eso se expresa en la tabla 2 .

El coeficiente de correlación entre las variables peso y talla es de 0.55 , lo que indica una correlación moderada entre ambas variables.

Por otro lado se obtuvo una alta correlación entre las variables peso y circunferencia de cintura, debido a que los valores obedecen a una tendencia lineal. El coeficiente de correlación entre ambas variables fue 0,87 , lo que significa que son directamente proporcionales, es decir a medida que aumenta el peso aumenta la circunferencia de cintura.

La lectura del comportamiento de estas variables corresponde a que los escolares que presenten un peso elevado presentaran valores de circunferencia de cintura aumentados. Esta relación puede deberse a que incrementos de peso generan aumentos de los depósitos de grasa corporal, los cuales se encuentran principalmente a nivel abdominal, generando de esta forma un aumento en el perímetro de cintura.

El coeficiente de correlación entre el peso y el índice de masa corporal fue 0,86 lo que se clasifica como una alta correlación debido a que el índice de masa corporal se calcula a partir del peso, lo que justifica su proporcionalidad directa.

El coeficiente de correlación entre las variables talla y circunferencia de cintura fue 0,39 , lo que deja de manifiesto que ambas variables son independientes linealmente, lo que significa que no se correlaciona un aumento de los valores de circunferencia de cintura en adolescentes que presenten talla elevada, ya que principalmente la circunferencia de cintura se ve influenciada por otros factores como el peso.

La correlación de las variables talla e índice de masa corporal es igual a 0,047 (gráfico 1) por lo que se puede afirmar que ambas variables son independientes entre sí, ya que un valor igual a cero significa independencia absoluta entre las variables. Esta independencia se debe a que en el cálculo del índice de masa corporal, la cual es una fracción, el cociente es la talla al cuadrado, lo que hace que no exista dependencia lineal entre la talla y el índice de masa corporal, lo que no significa que exista otra relación no lineal.

Entre las variables, el índice de masa corporal y la circunferencia de cintura existe una alta correlación lineal, directamente proporcionales, ya que el valor del coeficiente de correlación corresponde a 0,80 (gráfico 1). Esta alta correlación puede deberse a que el índice de masa corporal refleja la composición corporal general, sin diferenciación de compartimientos, por lo que aumentos de este indicador antropométrico lleva a un incremento en la circunferencia de cintura, ya que esta medición corresponde a la evaluación de una sección corporal específica, como es el tejido adiposo abdominal el que se ve afectado al modificarse la composición corporal general.

\section{DISCUSIÓN}

La población infantil chilena presenta una tendencia creciente a la malnutrición por exceso, reforzada por las conductas alimentarias del niño, del núcleo familiar y del contexto sociocultural que refuerza el consumo de alimentos altamente calóricos. Por otro lado, la carencia de un control nutricional adecuado en poblaciones vulne-

TABLA 2

Correlaciones de variables cuantitativas.

\begin{tabular}{lccccc} 
& Edad & Peso & Talla & CC & IMC \\
\hline Edad & 1 & 0,196 & 0,040 & 0,168 & 0,205 \\
Peso & 0,196 & 1 & 0,550 & 0,872 & 0,856 \\
Talla & 0,040 & 0,550 & 1 & 0,386 & 0,047 \\
CC & 0,168 & 0,872 & 0,386 & 1 & 0,804 \\
IMC & 0,205 & 0,856 & 0,047 & 0,804 & 1
\end{tabular}


rables así como la no adherencia a las recomendaciones nutricionales efectuadas por profesionales influyen en los problemas señalados, sobre todo considerando que la Organización Mundial de la Salud (OMS) considera a la obesidad infantil como una enfermedad crónica, por el riesgo de perpetuarla durante la vida y presentar diabetes tipo 2 y cardiopatía isquémica arterioesclerótica en su vida adulta (14)

La circunferencia de la cintura es una medida antropométrica simple y efectiva que puede ayudar a tamizar la obesidad abdominal en adultos $(15,16)$ y en niños (17), y también puede ser un mejor indicador de riesgo de enfermedad cardiovascular, que el índice de masa corporal en adultos y en niños (18).

En niños, la circunferencia de la cintura es mejor indicador de grasa visceral que el índice de masa corporal (19), demostrándolo así la evidencia.

Varios autores aseveran que el Indice de Masa Corporal, sólo puede subestimar la proporción de obesidad (20-22) y que no es suficiente como indicador de riesgo de alteraciones metabólicas, porque no discrimina la masa grasa de la magra en un individuo $(23,24)$, por lo cual la evaluación antropométrica debería complementarse con la circunferencia de la cintura.

Las relaciones entre variables en la población estudiada demuestran la existencia de una concordancia de los resultados con la información bibliográfica disponible en la literatura que señala que el índice de Masa Corporal (IMC) es uno de los indicadores antropométricos que muestra la mejor correlación con la grasa corporal total, aun cuando esta fluctúa entre 0.32 y 0.90 dependiendo de la edad, sexo, raza y el estadio puberal alcanzado (25).

Al realizar el análisis fue posible demostrar que las variables, índice de masa corporal, circunferencia de cintura y peso, se pueden agrupar, lo que significa que un diagnóstico efectivo se debe realizar considerando estas tres variables, ya que la dependencia entre ellas es muy elevada. Es decir el riesgo cardiometabólico (circunferencia de cintura aumentada) debe estar relacionado con un diagnóstico de malnutrición por exceso (IMC).

El niño con obesidad central no recibe atención médica y nutricional especializada en los programas

\section{GRÁFICO 1}

Dispersión y correlación de variables antropométricas.
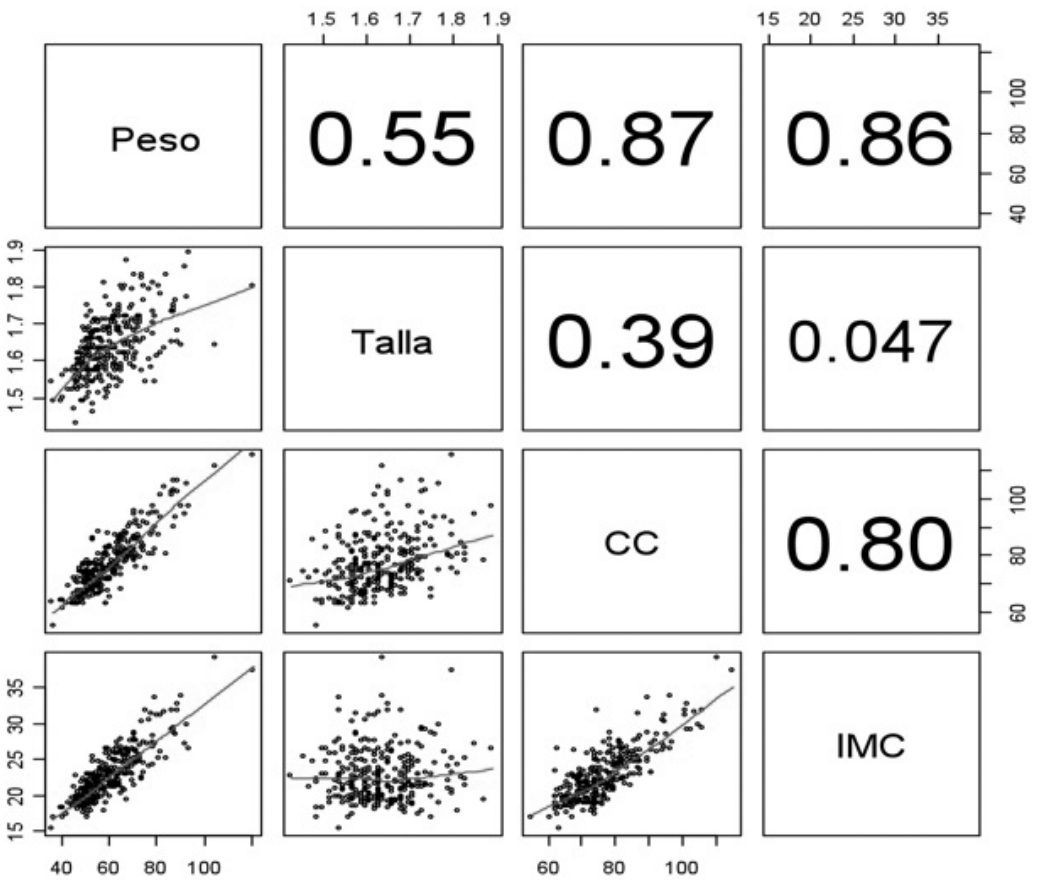
dirigidos al escolar, pues la obesidad todavía no se considera como indicador de atención prioritaria en salud. No obstante, es claro el hecho de que un niño con obesidad central, y posteriormente un adulto obeso, desarrollará una serie de patologías asociadas que van desde la resistencia a la insulina (diabetes mellitus tipo 2) hasta la enfermedad cardiovascular (26).

Se puede concluir que el síndrome metabólico posee una relación directa con ciertas variables que condicionan la presencia de patologías nutricionales, que en la práctica significa que los indicadores antropométricos pueden ser adecuados para identificar la presencia de riesgo cardiometabólico en adolescentes y con esto tomar las medidas terapéuticas, desde una perspectiva de anticipación al daño desde los escenarios de la promoción de la salud y prevención de la enfermedad hasta la curación, con el fin de evitar la enfermedad y sus secuelas.

\section{RESUMEN}

La malnutrición por exceso en el adolescente es un problema prevalente y aumenta el riesgo de desarrollar enfermedades metabólicas. El objetivo de este estudio fue asociar el síndrome metabólico y el riesgo cardiovascular en escolares de la comuna de Lota de Chile. Estudio de corte transversal, muestreo probabilístico aleatorio simple, se tomó una muestra de 286 niños, de 14 y 18 años de edad. Se estableció la prevalencia de malnutrición, y se realizó una correlación de Pearson. Las correlaciones fueron: peso y talla de 0.55 ; peso y circunferencia de cintura 0,87 ; el peso y el índice de masa corporal de 0,86 ; la talla y la circunferencia de cintura de 0,39 ; la talla e índice de masa corporal de 0,047 ; índice de masa corporal y circunferencia de cintura 0,80 . El índice de masa corporal, la circunferencia de cintura y el peso, se agrupan en un diagnóstico efectivo. El síndrome metabólico posee una relación que condiciona la presencia de patologías nutricionales, significa que los indicadores antropométricos pueden ser adecuados para identificar la presencia de riesgo cardiometabólico en adolescentes.

Palabras clave: Obesidad; sobrepeso; riesgo cardiometabólico; escolares; circunferencia de cintura.

Dirigir la correspondencia a:

Profesor

Kristian Buhring Bonacich, MSc

Departamento de Salud Pública

Facultad de Medicina

Universidad Católica de la Santísima Concepción

Alonso de Ribera 2850

Concepción, Chile
Teléfono: 56-41-2735416

Fax: 56-41-2735401

E mail: kbuhring@ucsc.cl

\section{BIBLIOGRAFÍA}

1. Organización Panamericana para la Salud, Oficina Regional para las Américas de la Organización Mundial para la Salud. Adolescencia. 2008.

2. Ruz O, Manuel y cols. Nutrición y Salud. Ed. Departamento de Nutrición- Facultad de Medicina. Universidad de Chile. P 213. 1996.

3. Cattani O., Andreína. Pubertad normal. Manual de pediatría 2006 Disponible en dirección LRL. htp./lescmela med pucelppaginas/publicaciones/ manualped/Pubertad.html.

4. Gobierno de Chile. Ministerio de Salud. Población Censo 2002 por servicios de salud. Departamento de estadística e información en salud. 2003.

5. Gobierno de Chile. Ministerio de Salud. Situación Nutricional del preescolar, escolar y adolescente. Epidemiología nutricional. 2004.

6. Escobar F., María Cristina y cols. Programa de Salud Cardiovascular. Ed. Ministerio de Salud. Chile. P 5, 2002.

7. Savva S, Tornaritis M, Savva M, Kourides Y, Panagi A, Silikiotou N, et al. Waist circumference and waist-to-height ratio are better predictors of cardiovascular disease risk factors in children than body mass index. Int J Obes Relat Metab Disord 2000; 24(11):1453-8.

8. Sung R, Yu C, Choi K, McManus A, Li A, Xu S, et al. Waist circumference and body mass index in Chinese children: cutoff values for predicting cardiovascular risk factors. Int J Obes 2007; 31(3):550-8.

9. Piazza, Norma. La circunferencia de cintura en los niños y adolescentes. Arch Argent Pediatr 2005; 103 (1): 5-6.

10. Gobierno de Chile. Ministerio de Salud. "Guía educativa para una vida saludable". Guías alimentarías, Actividad física y tabaco. Estrategia de intervención nutricional. 2005.

11. Pizarro, Tito. Rodríguez, Lorena. Benavides, Xenia. Norma técnica de evaluación nutricional del niño de 6 a 18 años. Ed. Ministerio de Salud. p 1-20, 2003.

12. Barrera A. M. Gladys. Indicadores y referentes para Evaluación del estado nutritivo, crecimiento y riesgo metabólico. Ed. INTA, Universidad de Chile y Universidad de las Naciones Unidas. 2006.

13. Fernández, J, Redden D, Pietrobelli A, Allison D. Waist circumference percentiles in nationally representative samples of African-American, European- 
American, and Mexican-American children and adolescents. J Pediatr 2004; 145, (4), 439-44.

14. Chen CH, Dietz W Ed. Obesity in childhood and adolescence. Lippincott Williams and Wilkins. Philadelphia. Nestlé Nutrition Workshop Series. Pediatrics Program. 2002; V 49; p 63-82.

15. Bonora E, Micciolo R, Ghiatas AA, Lancaster JL, Alyassin A, Muggeo M, et al. Is it possible to derive a reliable estimate of human visceral and subcutaneous abdominal adipose tissue from simple anthropometric measurements? Metabolism 1995; 44 (12): 1617-25.

16. Pouliot MC, Despres JP, Lemieux S, Moorjani S, Bouchard C, Tremblay A, et al. Waist circumference and abdominal sagittal diameter: best simple anthropometric indexes of abdominal visceral adipose tissue accumulation and related cardiovascular risk in men and women. Am J Cardiol 1994; 73 (7): 460-8.

17. Taylor RW, Jones IE, Williams SM, Goulding A. Evaluation of waist circumference, waist-to-hip ratio, and the conicity index as screening tools for high trunk fat mass, as measured by dual-energy X-ray absorptiometry, in children aged 3-19 y. Am J Clin Nutr 2000; 72 (2): 490-5.

18. Savva SC, Tornaritis M, Savva ME, Kourides Y, Panagi A, Silikiotou N, et al. Waist circumference and waist-to-height ratio are better predictors of cardiovascular disease risk factors in children than body mass index. Int J Obes Relat Metab Disord 2000; 24 (11): 1453-8.

19. Brambilla P, Bedogni G, Moreno LA, Goran MI, Gutin B, Fox KR, et al. Crossvalidation of anthropometry against magnetic resonance imaging for the assessment of visceral and subcutaneous adipose tissue in children. Int J Obes (Lond) 2006; 30 (1): 23-30.
20. Williams CL, Hayman LL, Daniels SR, Robinson TN, Steinberger J, Paridon S, et al. Cardiovascular health in childhood: a statement for health professionals from the Committee on Atherosclerosis, Hypertension, and Obesity in the Young (AHOY) of the Council on Cardiovascular Disease in the Young, American Heart Association. Circulation 2002; 106 (1): 143:60.

21. Ekelund U, Ong K, Linne Y, Neovius M, Brage S, Dunger DB, et al. Upward weight percentile crossing in infancy and early childhood independently predicts fat mass in young adults: the Stockholm Weight Development Study (SWEDES). Am J Clin Nutr 2006; 83 (2): 324-30.

22. Gustat J, Elkasabany A, Srinivasan S, Berenson GS. Relation of abdominal height to cardiovascular risk factors in young adults: the Bogalusa heart study. Am J Epidemiol 2000; 151 (9): 885-91.

23. Berenson GS, Srinivasan SR, Bao W, Newman WP, Tracy RE, Wattigney WA. Association between multiple cardiovascular risk factors and atherosclerosis in children and young adults. The Bogalusa Heart Study. N Engl J Med 1998; 338 (23): 1650-6.

24. Neovius M, Linne Y, Rossner S. BMI, waist-circumference and waist-hip-ratio as diagnostic tests for fatness in adolescents. Int J Obes (Lond) 2005; 29 (2): 163-9.

25. Díaz E, Burrows R, Muzzo S, Galgani J, Rodriguez R. Evaluación nutricional del adolescente mediante Indice de Masa Corporal (IMC) según etapa puberal. Rev Chil Pediatr 1996; 67(4):163-8.

26. María V. Benjumea R.,Dora I. Molina de S., Patricia E. Arbeláez B., Luz M. Agudelo G. Circunferencia de la cintura en niños y escolares manizaleños de 1 a 16 años. Rev Colombiana Cardiol 2008; 15 (1):23-24. 